Transiti on from di el ectri c barrier di scharge to mi crohol I ow di scharge i n paral I el - pl at e el ectrodes wi th i nt er cavi ty microhol es

\begin{tabular}{|l|l|}
\hline 著者 & Wat anabe Jun, Nagat su Nasaaki \\
\hline $\begin{array}{l}\text { j ournal or } \\
\text { publ i cat i on t i t l e }\end{array}$ & Appl i ed Physi cs Let t er s \\
\hline vol une & 98 \\
\hline number & 22 \\
\hline page r ange & 221502 \\
\hline year & $2011-05-31$ \\
\hline 出版者 & Amer i can I nst i t ut e of Physi cs \\
\hline 権利 & ( C) 2011 Aner i can I nst i t ut e of Physi cs \\
\hline URL & ht t p: //hdl . handl e. net /10297/5720 \\
\hline
\end{tabular}




\title{
Transition from dielectric barrier discharge to microhollow discharge in parallel-plate electrodes with intercavity microholes
}

\author{
Jun Watanabe and Masaaki Nagatsu ${ }^{\text {a) }}$ \\ Graduate School of Science and Technology, Shizuoka University, 3-5-1 Johoku, Naka-ku, \\ Hamamatsu 432-8561, Japan
}

(Received 2 February 2011; accepted 9 May 2011; published online 31 May 2011)

\begin{abstract}
Parallel-plate electrodes with microholes were used to study microhollow discharges (MHDs) triggered by dielectric barrier discharge (DBD) generated by applying a low-frequency high ac voltage under atmospheric conditions. The results obtained reveal that MHD is ignited simultaneously with spiky DBD signals generated by applying two cycles of time-truncated sinusoidal waves. The discharge ignition voltage of MHD was about $1 \mathrm{kV}$ lower for electrodes with intercavity holes than for electrodes with conventional straight holes. This reduction is due to the triggering effect of self-generated DBD. (C) 2011 American Institute of Physics.
\end{abstract}

[doi:10.1063/1.3595320]

Many studies have focused on microplasmas at atmospheric pressure including dielectric barrier discharge (DBD),${ }^{1-8}$ atmospheric pressure plasma jets, ${ }^{9-11}$ and microhollow cathode discharge (MHCD). ${ }^{12-14}$ High-density plasmas are desirable for performing fast material processing in industrial applications such as surface modification, film deposition, decomposition of gaseous and liquid pollutants, and inactivation of microorganisms. Of the three abovementioned methods, MHCDs generated using two parallelplate electrodes containing microholes are more suitable for generating high particle fluences because they generate longer discharges than DBD or atmospheric pressure plasma jets. Schoenbach et al. ${ }^{12-14}$ have investigated MHCD extensively, but their studies have mostly been limited to electrodes with a single microhollow, probably because it is difficult to sustain multihole MHCDs, especially in dc operation.

We previously investigated the discharge characteristics of dc MHCD using a parallel-plate electrode with $300 \mu \mathrm{m}$ diameter microholes and a $500 \mu \mathrm{m}$ long insulating space; this system was also equipped with additional DBD electrodes, which were used as a preionization source. ${ }^{15}$ With DBD preionization, we generated MHCD at a discharge ignition voltage of $2.4 \sim 2.5 \mathrm{kV}$, which is lower than that $(3.1 \sim 3.2 \mathrm{kV})$ without the DBD electrodes.

Therefore, in the present study, we apply high ac voltages directly to microhollow discharge (MHD) electrodes without using additional DBD electrodes. Here, we simply used the description of MHD in the presence or absence of hollow cathode effect. We investigate the transition from DBD to MHD triggered by self-generating DBD by using two parallel MHD electrodes with and without intercavities in the microholes. To study the temporal relation between the ignitions of MHD and DBD, we used two truncated cycles of a sinusoidal voltage followed by a $1 \mathrm{~s}$ off period.

Figure 1(a) shows the experimental setup for the MHD electrodes, which consist of two $100 \mu \mathrm{m}$ thick molybdenum sheets and a mica sheet as a spacer. We employed two kinds of electrodes: one is the conventional MHD electrodes with straight $300 \mu \mathrm{m}$ diameter through-holes and a $500 \mu \mathrm{m}$

${ }^{a)}$ Electronic mail: tmnagat@ipc.shizuoka.ac.jp. thick mica spacer [Fig. 1(b)] and the other is those with straight through-holes having an intercavity with a diameter of the mid-cavity in the insulating sheet, $D$, and a depth of $170 \mu \mathrm{m}$ for each hole [Fig. 1(c)]. Here, $D$ was varied from 0.3 to $10 \mathrm{~mm}$. In both the cases, we can expect that when the high truncated sinusoidal ac voltages were supplied between the electrodes, the DBD will generate inside the hole under atmospheric conditions (in air at $1 \mathrm{~atm}$ ). Especially, in the intercavity DBD electrodes, we can expect that the DBD will be more easily generated in the small gap region sandwiched between two insulating sheets, as illustrated in Fig. 1(c).

As mentioned above, MHD was generated by applying two cycles of a truncated ac high-voltage with of a sinusoidal wave with a period $T$ ranging from $100 \mu \mathrm{s}(10 \mathrm{kHz})$ to $10 \mathrm{~s}$ $(0.1 \mathrm{~Hz})$ followed by a $1 \mathrm{~s}$ off period between the electrodes. The voltage and current waveforms applied to the MHD electrodes were measured using a high-voltage divider and a

(a)

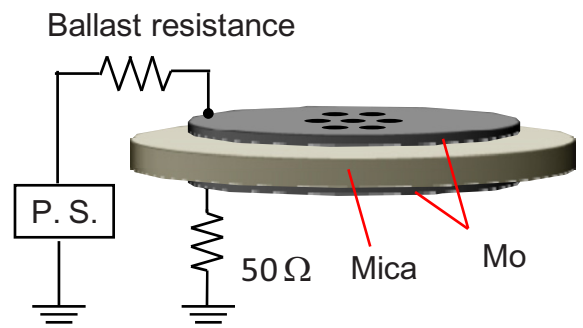

(b)

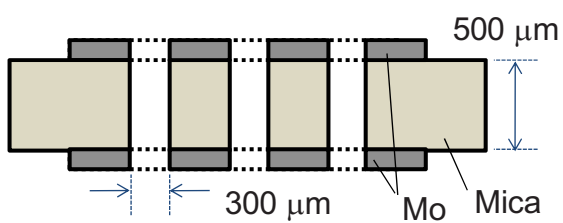

(c)

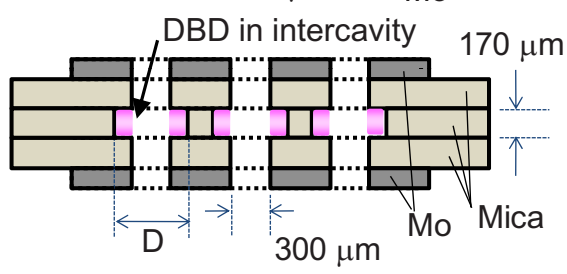

FIG. 1. (Color online) Schematic diagrams of the experimental setup: (a) MHD electrodes and circuit, (b) conventional MHD electrodes, and (c) intercavity MHD electrodes. 
(a)

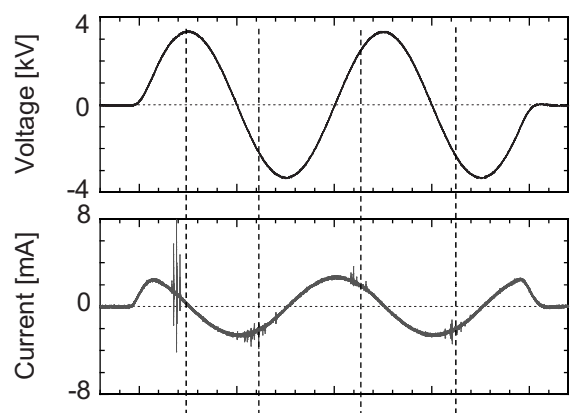

(b)


FIG. 2. Voltage and current waveforms for a conventional electrode with a single hole: (a) DBD and (b) MHD.

low-resistance $(50 \Omega)$ resistor and were recorded by a digital oscilloscope (LeCroy, 9314AM). We used a ballast resistance of $100 \mathrm{k} \Omega$.

In order to investigate the relation between ignition times of DBD and MHD, we first used a conventional electrode with only a single hole, which is different from an electrode with multiple holes shown in Fig. 1(b). The DBD currents were measured for different input signal frequencies in the range $0.1 \mathrm{~Hz}$ to $10 \mathrm{kHz}$ at a fixed applied voltage of $\pm 3.0 \mathrm{kV}$. DBD was generated at about $50 \mathrm{~Hz}$ and above and the DBD current increased with increasing frequency. To study the transition from DBD to MHD, we increased the input voltage slightly while keeping the frequency at $5 \mathrm{kHz}$, which is sufficiently high to generate DBD. Figure 2 shows typical voltage and current waveforms near discharge ignition driven by two cycles of $5 \mathrm{kHz}$ sinusoidal waves. Figures 2(a) and 2(b) show the waveforms obtained for typical DBD and stable MHD, respectively. Spiky current waveforms were observed for DBD in all four phases when the applied input voltage was approximately $2.0 \mathrm{kV}$, except for the first half cycle [Fig. 2(a)]. Figure 2(b) shows the stable current waveform obtained for MHD, where the voltage waveform exhibits sudden drops in all four phases due to MHD. Discharge occurs at the maximum applied voltage (about 3.2 $\mathrm{kV}$ ) in the first half cycle, whereas discharge occurs at about $2.1 \sim 2.2 \mathrm{kV}$ in the rising phase of the other three half cycles. The dashed lines in Fig. 2 indicate the temporal relation between MHD and spiky DBD signals. Except in the first phase, both kinds of discharge have similar timings. These results indicate that DBD triggers MHD in the second and subsequent half cycles. At some frequencies and applied voltages, MHD also occurs when DBD occurs in the rising phase before the maximum applied voltage in the first half cycle.

We next investigated the electrodes with an intercavity structure shown in Fig. 1(c). Figure 3 shows the dependence of the discharge ignition voltage of MHD driven by DBD at

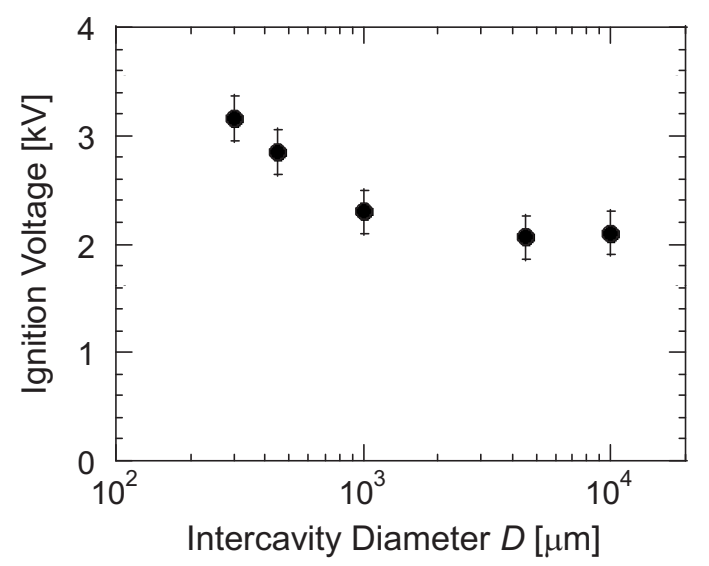

FIG. 3. Dependence of cavity diameter $D$ on the discharge ignition voltage of MHD at a fixed signal frequency of $1 \mathrm{kHz}$.

a fixed frequency of $1 \mathrm{kHz}$ on the mid-cavity diameter $D$. The discharge ignition voltage decreases with increasing $D$ and it almost saturates at $D \approx 1 \mathrm{~mm}$. Hence, we used a midcavity diameter of $D=4.5 \mathrm{~mm}$, which gives a discharge ignition voltage of about $2.0 \sim 2.2 \mathrm{kV}$.

Figure 4 shows the discharge waveforms for the intercavity electrode with a single hole. The input voltage has a frequency of $5 \mathrm{kHz}$. The DBD period is broader and the current peaks for DBD are higher in Fig. 4(a) than those in Fig. 2(a). Figure 4(b) shows that MHD occurs when DBD has discharge ignition voltages of $2.0 \mathrm{kV}$ or less. The discharge ignition voltages in the first half cycle $(\sim 2.0 \mathrm{kV})$ and subsequent half cycles $(1.2 \sim 1.6 \mathrm{kV})$ are significantly lower than those $(\sim 3.2 \mathrm{kV}$ and $2.1 \sim 2.2 \mathrm{kV}$, respectively) for conventional MHD electrodes.

Figure 5(a) shows a plot of the applied voltage for MHD ignition for conventional DBD electrodes with $0.3 \mathrm{~mm}$ diameter through-holes and intercavity DBD electrodes with $D$ $=4.5 \mathrm{~mm}$ as a function of the frequency of the input voltage. The rapid increase in the applied voltage with increasing

(a)


(b)

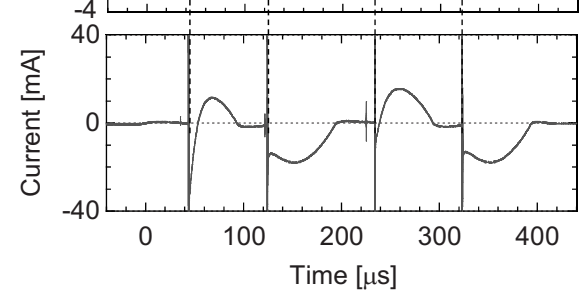

FIG. 4. Applied voltage and current waveforms for intercavity electrodes with a single hole: (a) DBD and (b) MHD. 

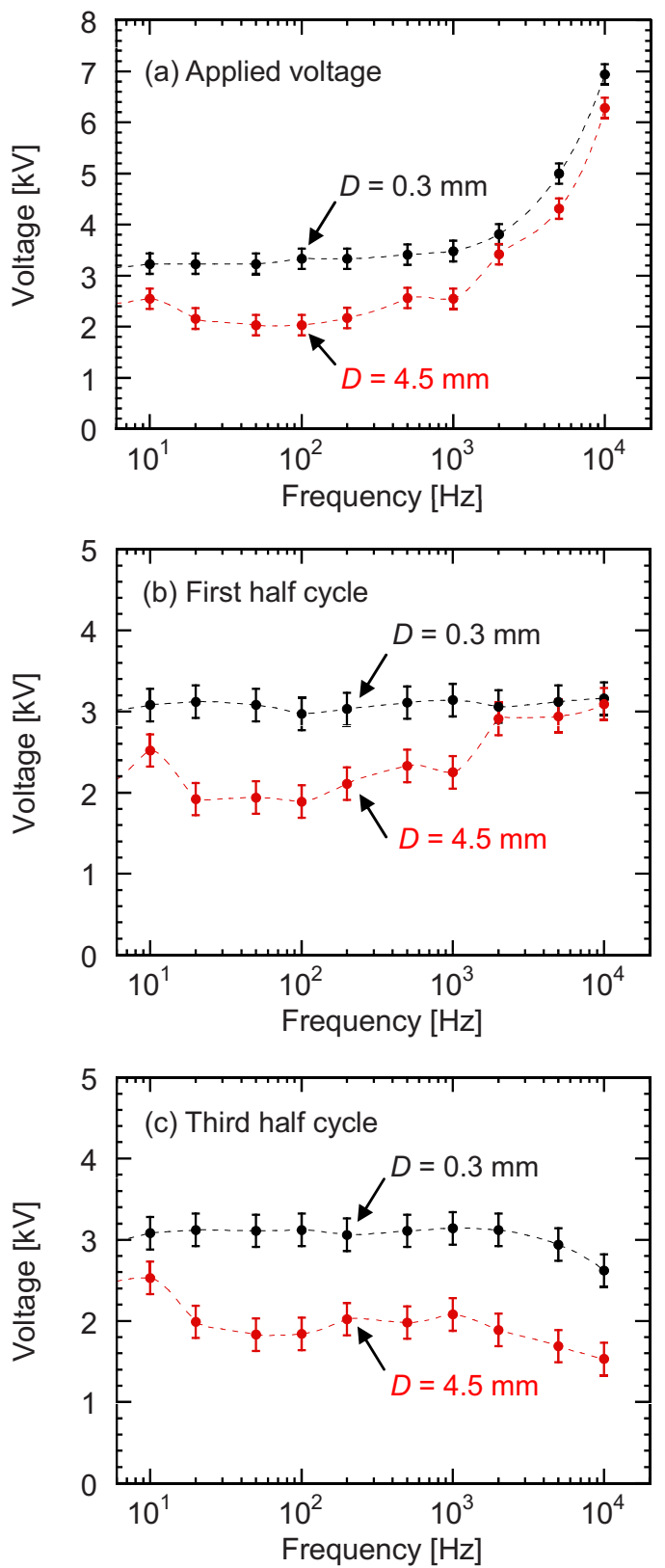

FIG. 5. (Color online) (a) Applied voltages at ignition of MHD, (b) MHD discharge ignition voltages at the first half cycle, and (c) those at the third half cycle in the cases of conventional $(D=0.3 \mathrm{~mm})$ and intercavity type $(D=4.5 \mathrm{~mm})$ DBD electrodes against the input sinusoidal wave frequency.

frequency is attributed to an increasing voltage drop at the ballast resistance due to the displacement current. The discharge ignition voltages in the first half cycle for the conventional electrodes are almost constant [see Fig. 5(b)]. However, the intercavity electrodes have a significantly lower discharge ignition voltage in the third half cycle than the conventional electrodes [see Fig. 5(c)]. This clearly indicates that DBD generated in the intercavity region can trigger MHD at a lower voltage because of the higher DBD current. Figure 5(b) shows that the discharge ignition voltages in the first half cycle for the intercavity electrode are comparable to those for the conventional electrode at frequencies higher than about $2 \mathrm{kHz}$; nevertheless, the discharge ignition voltages decrease due to DBD. This may be due to the applied sinusoidal voltage varying too rapidly at frequencies above $2 \mathrm{kHz}$ to cause a transition from DBD to MHD at a lower voltage than that for conventional electrodes. Comparison of the conventional and intercavity electrodes in the third half cycle clearly shows that the discharge ignition voltage is reduced by about $1.0 \mathrm{kV}$ in the intercavity MHD electrode at frequencies above $20 \mathrm{~Hz}$ [see Fig. 5(c)]. The results for the second half cycle (not shown) are similar to those for the third half cycle. The reduction in the discharge ignition voltages in the second and subsequent half cycles is attributed to residues (e.g., electrostatic charges on the surface of dielectric hole and excited neutral species) produced by the MHD plasma in the first half cycle.

Finally, we carried out the measurement of the discharge characteristics of multihole microhollow electrodes with 10 holes and compared them with conventional and intercavity DBD electrodes. Experimental result shows that all the holes of conventional electrodes with $300 \mu \mathrm{m}$ diameter straight holes were uniformly ignited by $1 \mathrm{kHz}$ ac voltages of $4.1 \mathrm{kV}$. In contrast, uniform MHDs occurred in all the holes of the intercavity electrodes with a cavity diameter of $D=10 \mathrm{~mm}$ at $2.47 \mathrm{kV}$, which was about $1.6 \mathrm{kV}$ lower than those for conventional electrodes. This is because the DBD generated in the cavity was strong enough to successively ignite MHD at a lower applied voltage than for the conventional electrodes.

In summary, we presented experimental results for the transition to MHD triggered by self-generated DBD at lowfrequency high ac voltages using parallel-plate electrodes containing microholes under atmospheric conditions. The results reveal that MHD occurred simultaneously with spiky DBD signals generated by low-frequency high ac voltages. As a result of triggering of self-generated DBD, the discharge ignition voltages for the intercavity electrodes were significantly lower (about $1 \mathrm{kV}$ in the single hole and about $1.6 \mathrm{kV}$ in the case of 10 microholes) than those for conventional through-hole electrodes.

The authors would like to thank Associate Professor A. Ogino of Shizuoka University for assisting with the data processing.

${ }^{1}$ U. Kogelschatz, Plasma Chem. Plasma Process. 23, 1 (2003).

${ }^{2}$ S. Kanazawa, M. Kogoma, T. Moriwaki, and S. Okazaki, J. Phys. D: Appl. Phys. 21, 838 (1988).

${ }^{3}$ S. Okazaki, M. Kogoma, M. Uehara, and Y. Kimura, J. Phys. D: Appl. Phys. 26, 889 (1993).

${ }^{4}$ F. Massines, A. Rabehi, P. Decomps, R. B. Gadri, P. Segur, and C. Mayoux, J. Appl. Phys. 83, 2950 (1998).

${ }^{5}$ D. Korzec, E. G. Finantu-Dinu, G. L. Dinu, J. Engemann, M. Stefecka, and M. Kando, Surf. Coat. Technol. 174-175, 503 (2003).

${ }^{6}$ O. Sakai, Y. Kishimoto, and K. Tachibana, J. Phys. D: Appl. Phys. 38, 431 (2005).

${ }^{7}$ B. Eliasson and U. Kogelschatz, Appl. Phys. B: Lasers Opt. 46, 299 (1988).

${ }^{8}$ S.-J. Park, K.-F. Chen, N. P. Ostrom, and J. G. Eden, Appl. Phys. Lett. 86, 111501 (2005)

${ }^{9}$ A. Schutze, J. Y. Jeong, S. E. Babayan, J. Park, G. S. Selwyn, and R. F. Hicks, IEEE Trans. Plasma Sci. 26, 1685 (1998).

${ }^{10}$ M. Teschke, J. Kedzeierski, E. G. Finantu-Dinu, D. Korzec, and J. Engemann, IEEE Trans. Plasma Sci. 33, 310 (2005).

${ }^{11}$ B. L. Sands, B. N. Ganguly, and K. Tachibana, Appl. Phys. Lett. 92, 151503 (2008).

${ }^{12}$ K. H. Schoenbach, A. El-Habachi, W. Shi, and M. Ciocca, Plasma Sources Sci. Technol. 6, 468 (1997).

${ }^{13}$ R. H. Stark and K. H. Schoenbach, Appl. Phys. Lett. 74, 3770 (1999).

${ }^{14}$ K. H. Schoenbach, M. Moselhy, A. Shi, and R. Bentley, J. Vac. Sci. Technol. A 21, 1260 (2003).

${ }^{15}$ J. Watanabe, A. Ogino, and M. Nagatsu, Appl. Phys. Lett. 91, 221507 (2007) 